\title{
AP17-OLR Challenge: Data, Plan, and Baseline
}

\author{
Zhiyuan Tang*, Dong Wang*, Yixiang Chen* and Qing Chen ${ }^{\dagger}$ \\ * Center for Speech and Language Technologies, Tsinghua University \\ Corresponding author: wangdong99@mails.tsinghua.edu.cn \\ $\dagger$ SpeechOcean \\ E-mail: chenqing@speechocean.com
}

\begin{abstract}
We present the data profile and the evaluation plan of the second oriental language recognition (OLR) challenge AP17-OLR. Compared to the event last year (AP16-OLR), the new challenge involves more languages and focuses more on short utterances. The data is offered by SpeechOcean and the NSFC M2ASR project. Two types of baselines are constructed to assist the participants, one is based on the i-vector model and the other is based on various neural networks. We report the baseline results evaluated with various metrics defined by the AP17-OLR evaluation plan and demonstrate that the combined database is a reasonable data resource for multilingual research. All the data is free for participants, and the Kaldi recipes for the baselines have been published online.
\end{abstract}

\section{INTRODUCTION}

Oriental languages can be divided into several language families, including Austroasiatic languages (e.g., Vietnamese, Cambodia ) [1], Tai-Kadai languages (e.g., Thai, Lao), Hmong-Mien languages (e.g., some dialects in south China), Sino-Tibetan languages (e.g., Chinese Mandarin), Altaic languages (e.g., Korea, Japanese), Indo-European languages (e.g., Russian) [2], [3], [4]. The increasing demographic migration and international business interaction have caused rich multilingual phenomena, e.g., codes switching from a primary language to a second language, and then switching back, where the second language part is just one or two words. Due to the geographical proximity, oriental languages influence each other, resulting in complex development patterns in terms of both phonetics and linguistics. These complex patterns make the multilingual research on these languages particularly attractive.

To meet the request of this research, the center for speech and language technologies (CSLT) at Tsinghua University and SpeechOcean organized the first oriental language recognition (OLR) challenge during APSIPA ASC 2016 [5]. The goal of the challenge was to demonstrate how the state-of-art language identification (LID) techniques can discriminate oriental languages, as well as fostering new technologies in this direction. This challenge was very successful: 8 teams from China mainland, Taiwan, Singapore, and Germany submitted 9 submissions, and the best system submitted by the NUSI2R joint team achieved very good performance $\left(C_{a v g}=0.0113\right.$, EER\%=1.09). This result partly demonstrated that with the present technology, it is possible to discriminate the typical oriental languages from each other, at least under the conditions set by the challenge. More details can be found from the challenge web site 1

Encouraged by the success of AP16-OLR, we propose the second OLR challenge, based on APSIPA ASC 2017. This new challenge, denoted by AP17-OLR, will involve more languages and thus be more challenging. Besides the 7 languages in AP16-OLR, 3 new oriental languages are involved: Uyghur, Kazakh and Tibetan. Uyghur and Kazakh belong to the Turkic language, and Tibetan belongs to the Sino-Tibetan language. Both are major minority languages in China and are spoken by relatively large populations. The data is offered by the NSFC M2ASR project following the M2ASR Free Data Program.

Another feature of the new challenge is that we put more emphasis on short utterances. Since the performance on long utterances had been demonstrated in AP16-OLR, we start to tackle the more challenging scenario with short utterances. More importantly, LID on short utterances can find significant practical values, for example in speech recognition with codeswitching. Unfortunately, the pervious state-of-the-art i-vector model is not very suitable in the short-utterance scenario, as it heavily relies on the distributional patterns of the acoustic features, for which a long utterance is necessary. Recently, researchers found that methods based on deep neural models potentially solve this problem. This neural approach utilizes various forms of deep neural networks (DNNs) to discover language sensitive features from short-term segments [6], [7], [8], [9], [10], [11], [12], [13]. To meet the practical request and reflect the recent research advantage, AP17-OLR moves its focus to short utterances, e.g., 1 second or 3 seconds.

In the rest of the paper, we will present the data profile and the evaluation plan of the AP17-OLR challenge. To assist participants to build their own submissions, two types of baseline systems are constructed, based on the i-vector model and various DNN models respectively. The Kaldi recipes of these baselines can be downloaded from the challenge web site.

\section{DATABASE PROFILE}

Participants of AP17-OLR can request the following datasets for system construction.

- AP16-OL7: The standard database for AP16-OLR, including AP16-OL7-train, AP16-OL7-dev, and AP16OL7-test.

\footnotetext{
${ }^{1}$ http://olr.cslt.org

${ }^{2}$ http://m2asr.cslt.org
} 
TABLE I

AP17-OL3 AND AP16-OL7 DATA PROFILE

\begin{tabular}{|c|c|c|c|c|c|c|c|c|}
\hline \multicolumn{3}{|c|}{ AP17-OL3 } & \multicolumn{3}{|c|}{ AP17-OL3-train/dev } & \multicolumn{3}{|c|}{ AP17-OL3-test } \\
\hline Code & Description & Channel & No. of Speakers & Utt./Spk. & Total Utt. & No. of Speakers & Utt./Spk. & Total Utt. \\
\hline ka-cn & Kazakh in China & Mobile & 86 & 50 & 4200 & 86 & 20 & 1800 \\
\hline ti-cn & Tibetan in China & Mobile & 34 & 330 & 11100 & 34 & 50 & 1800 \\
\hline uy-id & Uyghur in China & Mobile & 353 & 20 & 5800 & 353 & 5 & 1800 \\
\hline \multicolumn{3}{|c|}{ AP16-OL7 } & \multicolumn{3}{|c|}{ AP16-OL7-train/dev } & \multicolumn{3}{|c|}{ AP16-OL7-test } \\
\hline Code & Description & Channel & No. of Speakers & Utt./Spk. & Total Utt. & No. of Speakers & Utt./Spk. & Total Utt. \\
\hline ct-cn & Cantonese in China Mainland and Hongkong & Mobile & 24 & 320 & 7559 & 6 & 300 & 1800 \\
\hline zh-cn & Mandarin in China & Mobile & 24 & 300 & 7198 & 6 & 300 & 1800 \\
\hline id-id & Indonesian in Indonesia & Mobile & 24 & 320 & 7671 & 6 & 300 & 1800 \\
\hline ja-jp & Japanese in Japan & Mobile & 24 & 320 & 7662 & 6 & 300 & 1800 \\
\hline ru-ru & Russian in Russia & Mobile & 24 & 300 & 7190 & 6 & 300 & 1800 \\
\hline ko-kr & Korean in Korea & Mobile & 24 & 300 & 7196 & 6 & 300 & 1800 \\
\hline vi-vn & Vietnamese in Vietnam & Mobile & 24 & 300 & 7200 & 6 & 300 & 1800 \\
\hline
\end{tabular}

Male and Female speakers are balanced.

The number of total utterances might be slightly smaller than expected, due to the quality check.

- AP17-OL3: A new dataset provided by the M2ASR project, involving three new languages. It contains AP17OL3-train and AP17-OL3-dev.

- THCHS30: The THCHS30 database (plus the accompanied resources) published by CSLT, Tsinghua University [14].

Participants can use all the above data to train their submission systems, but are required to report the results on the development set. The development set includes AP16-OL7-test and AP17-OL3-dev. Note that the development data should be excluded from the training data when constructing the presubmission system and computing the dev results.

Besides the speech signals, the AP17-OL3 and AP16OL7 databases also provide lexicons of all the 10 languages, as well as the transcriptions of all the training utterances. These resources allow training acoustic-based or phoneticbased language recognition systems. Training phone-based speech recognition systems is also possible, though large vocabulary recognition systems are not well supported, due to the lack of large-scale language models.

A test dataset will be provided at the date of result submission. This test set involves two parts: AP17-OL3-test and AP17-OL7-test. The latter involves 7 languages that is the same as in AP16-OL7. The details of these databases are described as follows.

\section{A. AP16-OL7}

The AP16-OL7 database was originally created by Speechocean targeting for various speech processing tasks. It was provided as the standard training and test data in the AP16OLR challenge. The entire database involves 7 datasets, each in a particular language. The seven languages are: Mandarin, Cantonese, Indoesian, Japanese, Russian, Korean and Vietnamese. The data volume for each language is about 10 hours of speech signals recorded in reading style. The signals were recorded by mobile phones, with a sampling rate of $16 \mathrm{kHz}$ and a sample size of 16 bits.

For Mandarin, Cantonese, Vietnamese and Indonesia, the recording was conducted in a quiet environment. As for
Russian, Korean and Japanese, there are 2 recording sessions for each speaker: the first session was recorded in a quiet environment and the second was recorded in a noisy environment. The basic information of the AP16-OL7 database is presented in Table I. and the details of the database can refer to the AP16-OLR challenge web site or the description paper [5].

\section{B. AP17-OL7-test}

The AP17-OL7 database is a new dataset provided by SpeechOcean. This dataset contains 7 languages as in AP16-OL7, each containing 1800 utterances. The recording conditions are the same as AP16-OL7. This database is used as part of the test set for the AP17-OLR challenge.

\section{AP17-OL3}

The AP17-OL3 database contains 3 languages: Kazakh, Tibetan and Uyghur, all are minority languages in China. This database is part of the Multilingual Minorlingual Automatic Speech Recognition (M2ASR), which is supported by the National Natural Science Foundation of China (NSFC). The project is a three-party collaboration, including Tsinghua University, the Northwest National University, and Xinjiang University. The aim of this project is to construct speech recognition systems for five minor languages in China (Kazakh, Kirgiz, Mongolia, Tibetan and Uyghur). However, our ambition is beyond that scope: we hope to construct a full set of linguistic and speech resources and tools for the five languages, and make them open and free for research purposes. We call this the M2ASR Free Data Program. All the data resources, including the tools published in this paper, are released on the web site of the project.

The sentences of each language in AP17-OL3 are randomly selected from the original M2ASR corpus. The data volume for each language in AP17-OL3 is about 10 hours of speech signals recorded in reading style. The signals were recorded by mobile phones, with a sampling rate of $16 \mathrm{kHz}$ and a sample size of 16 bits. We selected 1800 utterances for each language as the development set (AP17-OL3-dev), and the rest is used as the training set (AP17-OL3-train). The test set of each language involves 1800 utterances, and is provided separately and 
denoted by AP17-OL3-test. Compared to AP16-OL7, AP17OL3 contains much more variations in terms of recording conditions and the number of speakers, which may inevitably increase the difficulty of the challenge task. The information of the AP17-OL3 database is summarized in Table I

\section{AP17-OLR CHALLENGE}

Based on the experience of AP16-OLR challenge, we call the AP17-OLR challenge ${ }^{3}$ Following the definition of NIST LRE15 [15], the task of the challenge is defined as follows: Given a segment of speech and a language hypothesis (i.e., a target language of interest to be detected), the task is to decide whether that target language was in fact spoken in the given segment (yes or no), based on an automated analysis of the data contained in the segment. The evaluation plan mostly follows the principles of NIST LRE15. It focuses on the closeset condition, i.e., the language identification task.

The challenge focuses on short utterances. We define three test conditions according to the length of the test utterances: 1 second condition, 3 second condition and full-utterance condition. The test utterances of the 1 second condition and 3 second condition are randomly excerpted from the original ones. If a test utterance is not sufficient long for the excerption, it is simply discarded. The evaluation details are described as follows.

\section{A. System input/output}

The input to the LID system is a set of speech segments in unknown languages (but within the 10 languages of AP17OL3 and AP16-OL7). The task of the LID system is to determine the confidence that a language is contained in a speech segment. More specifically, for each speech segment, the LID system outputs a score vector $<\ell_{1}, \ell_{2}, \ldots, \ell_{10}>$, where $\ell_{i}$ represents the confidence that language $i$ is spoken in the speech segment. Each score $\ell_{i}$ will be interpreted as follows: if $\ell_{i} \geq 0$, then the decision would be that language $i$ is contained in the segment, otherwise it is not. The scores should be comparable across languages and segments. This is consistent with the principle of LRE15, but differs from that of LRE09 [16] where an explicit decision is required for each trial.

In summary, the output of an OLR submission will be a text file, where each line contains a speech segment plus a score vector for this segment, e.g.,

$\begin{array}{cccccccc}\operatorname{seg}_{1} & 0.5 & -0.2 & -0.3 & \ldots & 0.1 & -9.2 & -0.1 \\ \operatorname{seg}_{2} & -0.1 & -0.3 & 0.5 & \ldots & 0.3 & -0.5 & -0.9 \\ \ldots & & & & \ldots & & & \end{array}$

\section{B. Test condition}

- No additional training materials, except AP17-OL3, AP16-OL7 and THCHS30, are allowed to be used.

- All the trials should be processed. Scores of lost trials will be interpreted as -inf.

$\sqrt[3]{\text { http://cslt.riit.tsinghua.edu.cn/mediawiki/index.php/OLR_Challenge_2017 }}$
- The speech segments in each test condition (1 second, 3 second or the full-utterance) should be processed independently, and each test segment in a group should be processed independently too. Knowledge from other test segments is not allowed to use (e.g., score distribution of all the test segments).

- Information of speakers is not allowed to use.

- Listening to any speech segments is not allowed.

\section{Evaluation metrics}

As in LRE15, the AP17-OLR challenge chooses $C_{a v g}$ as the principle evaluation metric. First define the pair-wise loss that composes the missing and false alarm probabilities for a particular target/non-target language pair:

$C\left(L_{t}, L_{n}\right)=P_{\text {Target }} P_{\text {Miss }}\left(L_{t}\right)+\left(1-P_{\text {Target }}\right) P_{F A}\left(L_{t}, L_{n}\right)$

where $L_{t}$ and $L_{n}$ are the target and non-target languages, respectively; $P_{M i s s}$ and $P_{F A}$ are the missing and false alarm probabilities, respectively. $P_{\text {target }}$ is the prior probability for the target language, which is set to 0.5 in the evaluation. Then the principle metric $C_{a v g}$ is defined as the average of the above pair-wise performance:

$$
C_{\text {avg }}=\frac{1}{N} \sum_{L_{t}}\left\{\begin{array}{l}
P_{\text {Target }} \cdot P_{\text {Miss }}\left(L_{t}\right) \\
+\sum_{L_{n}} P_{\text {Non-Target }} \cdot P_{F A}\left(L_{t}, L_{n}\right)
\end{array}\right\}
$$

where $N$ is the number of languages, and $P_{\text {Non-Target }}=$ $\left(1-P_{\text {Target }}\right) /(N-1)$. We have provided the evaluation script for system development.

\section{BASELINE SYSTEMS}

We constructed two kinds of baseline LID systems, based on the i-vector model and various DNN models respectively. All the experiments were conducted with Kaldi [17]. The purpose of these experiments is not to present a competitive submission, instead to present a reference for the participants. The recipes can be downloaded from the challenge web site.

\section{A. $i$-vector system}

The i-vector baseline systems were constructed based on the i-vector model [18], [19]. The static acoustic features involved 19-dimensional Mel frequency cepstral coefficients (MFCCs) and the log energy. This static features were augmented by their first and second order derivatives, resulting in 60-dimensional feature vectors. The UBM involved 2, 048 Gaussian components and the dimensionality of the i-vectors was 400. Linear discriminative analysis (LDA) was employed to promote language-related information. The dimensionality of the LDA projection space was set to 6 .

With the i-vectors (either original or after LDA transform), the score of a trail on a particular language can be simply computed as the cosine distance between the test i-vector and the mean i-vector of the training segments that belong to that language. This is denoted to be 'cosine distance scoring'. 
TABLE II

$\mathrm{C}_{a v g}$ AND EER RESULTS OF VARIOUS I-VECTOR BASELINE SYSTEMS ON 3 TEST CONDITIONS

\begin{tabular}{|c|c|c|c|c|c|c|}
\hline & \multicolumn{2}{|c|}{1 second } & \multicolumn{2}{|c|}{3 second } & \multicolumn{2}{|c|}{ Full-Length } \\
\hline System & $C_{a v g}$ & EER\% & $C_{a v g}$ & EER\% & $C_{a v g}$ & EER\% \\
\hline i-vector & 0.1672 & 15.28 & $\overline{0.0695}$ & 7.59 & 0.0522 & 6.224 \\
\hline i-vector + LDA & 0.1238 & 13.30 & 0.0494 & 5.95 & 0.0362 & 4.704 \\
\hline $\begin{array}{l}\text { i-vector } \\
\text { (Linear SVM) }\end{array}$ & 0.1485 & 14.43 & 0.0624 & 6.07 & 0.0469 & 4.58 \\
\hline $\begin{array}{l}\text { i-vector } \\
\text { (Poly SVM) }\end{array}$ & 0.1242 & 12.43 & 0.0470 & 4.83 & 0.0351 & 3.58 \\
\hline $\begin{array}{l}\text { i-vector } \\
\text { (RBF SVM) }\end{array}$ & 0.1313 & 12.16 & 0.0495 & 4.59 & 0.0352 & 3.39 \\
\hline $\begin{array}{l}\text { L-vector + LDA } \\
\text { (Linear SVM) }\end{array}$ & 0.1336 & 12.47 & 0.0492 & 4.74 & 0.0337 & 3.30 \\
\hline $\begin{array}{l}\text { L-vector + LDA } \\
(\text { Poly SVM) }\end{array}$ & 0.1415 & 12.98 & 0.0538 & 4.92 & 0.0373 & 3.49 \\
\hline $\begin{array}{l}\text { L-vector + LDA } \\
(\mathrm{RBF} \text { SVM) }\end{array}$ & 0.1370 & 12.61 & 0.0513 & 4.73 & 0.0355 & 3.32 \\
\hline
\end{tabular}

TABLE III

$\mathrm{C}_{a v g}$ AND EER RESULTS OF VARIOUS DNN BASELINE SYSTEMS ON 3 TEST CONDITIONS.

\begin{tabular}{|c|c|c|c|c|c|c|c|c|c|c|c|c|}
\hline & \multicolumn{4}{|c|}{1 second } & \multicolumn{4}{|c|}{3 second } & \multicolumn{4}{|c|}{ Full-Length } \\
\hline & \multicolumn{2}{|c|}{$C_{a v g}$} & \multicolumn{2}{|c|}{ EER\% } & \multicolumn{2}{|c|}{$C_{a v g}$} & \multicolumn{2}{|c|}{ EER\% } & \multicolumn{2}{|c|}{$C_{a v g}$} & \multicolumn{2}{|c|}{ EER\% } \\
\hline System & Fr. & Utt. & Fr. & Utt. & Fr. & Utt. & Fr. & Utt. & Fr. & Utt. & Fr. & Utt. \\
\hline$\overline{\text { TDNN-LID }}$ & 0.1930 & 0.1604 & 19.18 & 15.63 & 0.1920 & 0.1523 & 19.14 & 15.43 & 0.1925 & 0.1451 & 17.95 & 14.65 \\
\hline LSTM-LID & 0.1837 & 0.1569 & 19.13 & 16.77 & 0.1773 & 0.1525 & 18.90 & 16.99 & 0.1771 & 0.1468 & 17.87 & 16.03 \\
\hline PTN-LID & 0.1821 & 0.1153 & 18.43 & 11.88 & 0.1571 & 0.0727 & 16.03 & 8.24 & 0.1516 & 0.0689 & 14.82 & 8.15 \\
\hline
\end{tabular}

A more powerful scoring approach is to employ various discriminative models. In our experiment, we trained a support vector machine (SVM) for each language to determine the score that a test i-vector belongs to that language. The SVMs were trained on the $\mathrm{i}$-vectors of all the training segments, following the one-verse-rest scheme. We will call this scoring approach as 'SVM-based scoring'.

\section{B. DNN systems}

For the DNN baseline, three kinds of DNN architectures were designed. The first two are the traditional time-delay neural network (TDNN) [20] and recurrent neural network with long short-term memory units (LSTM-RNN) [21], as shown in Fig. 1 (a) and (b) respectively. The third one is based on the recently proposed phonetic temporal neural (PTN) model [22], where an auxiliary phonetic model produces phonetic feature, and an RNN LID model is used to identify the language. The architecture is shown in Fig. 1 (c). Both the the LSTM-RNN LID system and the PTN LID system employ an LSTM-RNN to identify languages; the difference is that the PTN system uses a phonetic model to extract phonetic features, rather than using raw acoustic features. Since the phonetic model is trained with a different objective function (phone discrimination), it can be seen as a mult-task training approach. In this study, the phonetic model is a TDNN and was trained using the THCHS30 database and the accompanied Kaldi recipe.

The raw feature of all the three DNN systems is 40dimensional Fbanks, with a symmetric 4-frame window for the TDNN and a symmetric 2-frame window for the LSTMRNN to splice neighboring frames. For the TDNN LID, there are 6 hidden layers, and the activation function is p-norm. The number of units of each TDNN layer is set to be 2048. The number of cells of the LSTM is set to be 1024 .

\section{Performance results}

The primary evaluation metric in AP17-OLR is $C_{a v g}$. Besides that, we also present the performance in terms of equal error rate (EER). These metrics evaluate system performance from different perspectives, offering a whole picture of the verification/identification capability of the tested system. The performance is evaluated on the development set at present.

The utterance level $\mathrm{C}_{a v g}$ and EER results of various ivector baseline systems are showed in Table III. The frame and utterance level $\mathrm{C}_{\text {avg }}$ and EER results of various DNN baseline systems are showed in Table III. From these results, we can observe that the i-vector systems generally perform well with long duration utterances. On short utterances, the PTN system performs the best. 
(a)

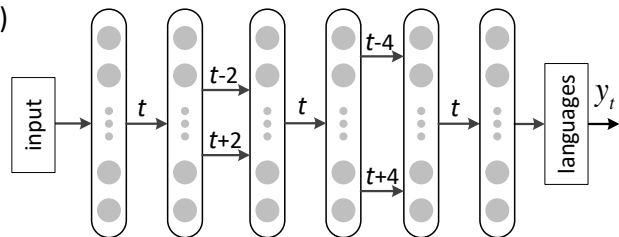

(b)

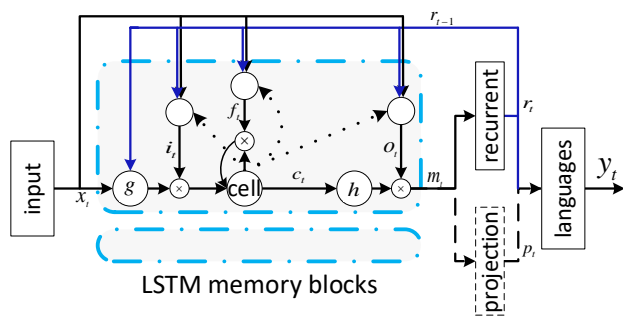

(c)

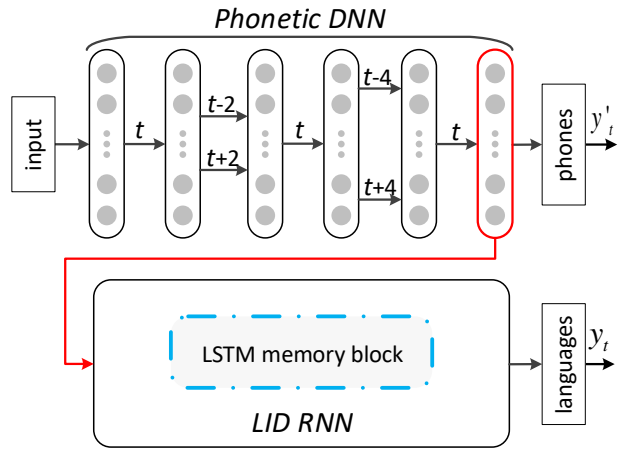

Fig. 1. Three neural-based LID architectures. The TDNN LID system (above), the LSTM-RNN LID system (middle) and the PTN LID system (bottom). In the PTN LID system, the phonetic feature is read from the last hidden layer of the phonetic DNN which is a TDNN and then becomes the only input for the system.

\section{CONCLUSiOns}

We presented the data profile and the evaluation plan of the AP17-OLR challenge. To assist participants to establish a reasonable starting system, we published two types of baseline systems based on the i-vector model and various DNN models respectively. All the data resources are free for the participants, and the recipes of the baseline systems can be freely downloaded.

\section{REFERENCES}

[1] P. Sidwell and R. Blench, "14 the austroasiatic urheimat: the southeastern riverine hypothesis," Dynamics of human diversity, p. 315, 2011.

[2] S. R. Ramsey, The languages of China. Princeton University Press, 1987.

[3] M. Shibatani, The languages of Japan. Cambridge University Press, 1990.

[4] B. Comrie, G. Stone, and M. Polinsky, The Russian language in the twentieth century. Oxford University Press, 1996.

[5] D. Wang, L. Li, D. Tang, and Q. Chen, "Ap16-ol7: A multilingual database for oriental languages and a language recognition baseline,' in APSIPA ASC. IEEE, 2016.

[6] I. Lopez-Moreno, J. Gonzalez-Dominguez, O. Plchot, D. Martinez, J. Gonzalez-Rodriguez, and P. Moreno, "Automatic language identification using deep neural networks," in Acoustics, Speech and Signal
Processing (ICASSP), 2014 IEEE International Conference on. IEEE 2014, pp. 5337-5341.

[7] J. Gonzalez-Dominguez, I. Lopez-Moreno, H. Sak, J. GonzalezRodriguez, and P. J. Moreno, "Automatic language identification using long short-term memory recurrent neural networks." in Interspeech, 2014, pp. 2155-2159.

[8] G. Gelly, J.-L. Gauvain, V. Le, and A. Messaoudi, "A divide-andconquer approach for language identification based on recurrent neural networks," Interspeech 2016, pp. 3231-3235, 2016.

[9] R. Zazo, A. Lozano-Diez, J. Gonzalez-Dominguez, D. T. Toledano, and J. Gonzalez-Rodriguez, "Language identification in short utterances using long short-term memory (LSTM) recurrent neural networks," PloS one, vol. 11, no. 1, p. e0146917, 2016

[10] A. Lozano-Diez, R. Zazo Candil, J. González Domínguez, D. T. Toledano, and J. Gonzalez-Rodriguez, "An end-to-end approach to language identification in short utterances using convolutional neural networks," in Proceedings of the Annual Conference of the International Speech Communication Association, INTERSPEECH. International Speech and Communication Association, 2015.

[11] M. Jin, Y. Song, I. Mcloughlin, L.-R. Dai, and Z.-F. Ye, "LID-senone extraction via deep neural networks for end-to-end language identification," in Proc. of Odyssey, 2016.

[12] M. Kotov and M. Nastasenko, "Language identification using time delay neural network d-vector on short utterances," in Speech and Computer. 18th International Conference, SPECOM 2016, Budapest, Hungary, August 23-27, 2016, Proceedings, vol. 9811. Springer, 2016, p. 443.

[13] D. Garcia-Romero and A. McCree, "Stacked long-term tdnn for spoken language recognition," Interspeech 2016, pp. 3226-3230, 2016.

[14] D. Wang and X. Zhang, "Thchs-30: A free chinese speech corpus," arXiv preprint arXiv:1512.01882, 2015.

[15] "The 2015 NIST language recognition evaluation plan (LRE15)," NIST 2015, ver. $22-3$.

[16] “The 2009 NIST language recognition evaluation plan (LRE09)," NIST, 2009, ver. 6

[17] D. Povey, A. Ghoshal, G. Boulianne, L. Burget, O. Glembek, N. Goel, M. Hannemann, P. Motlicek, Y. Qian, and P. Schwarz, "The kaldi speech recognition toolkit," in Proceedings of IEEE 2011 workshop on Automatic Speech Recognition and Understanding, no. EPFL-CONF192584. IEEE Signal Processing Society, 2011.

[18] N. Dehak, P. G. Kenny, R. Dehak, P. Dumouchel, and P. Ouellet, "Front-end factor analysis for speaker verification," IEEE Transactions on Audio, Speech, and Language Processing, vol. 19, no. 4, pp. 788798, 2011

[19] N. Dehak, P. A. Torres-Carrasquillo, D. A. Reynolds, and R. Dehak, "Language recognition via i-vectors and dimensionality reduction," in INTERSPEECH, 2011, pp. 857-860.

[20] K. J. Lang, A. H. Waibel, and G. E. Hinton, "A time-delay neura network architecture for isolated word recognition," Neural networks, vol. 3, no. 1, pp. 23-43, 1990.

[21] S. Hochreiter and J. Schmidhuber, "Long short-term memory," Neural computation, vol. 9, no. 8, pp. 1735-1780, 1997.

[22] Z. Tang, D. Wang, Y. Chen, L. Li, and A. Abel, "Phonetic temporal neural model for language identification," arXiv preprint arXiv:1705.03151, 2017. 